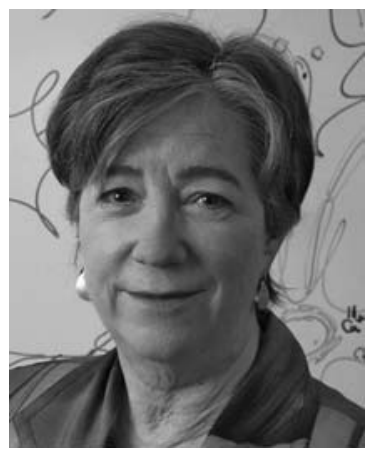

\title{
A Conversation with Susan Lindquist
}

\author{
INTERVIEWER: RICHARD SEVER \\ Executive Editor, CSH Perspectives and CSH Protocols
}

Susan Lindquist is a member of the Whitehead Institute for Biomedical Research and a Professor of Biology at the Massachusetts Institute of Technology.

Richard Sever: You work on yeast, but also on Alzheimer's disease and Parkinson's disease and various other conditions. Alzheimer's and Parkinson's are complex neurological conditions that affect multicellular organisms with nerves. Yeast is a single-celled organism with no nerves, so what's the logic behind that?

Dr. Lindquist: Sounds crazy, doesn't it? And believe me, we didn't have an easy time of it in the beginning. It was based upon the fact that I've been working on the protein folding problem for a very long time. And it's a problem that's universal amongst all living organisms. There are so many aspects of protein folding-protein trafficking, protein homeostasis - that are very highly conserved. So I thought it was worth a try.

We really have to solve these problems as their magnitude is staggering. As we've cured other diseases, we've been living longer but it's come at a terrible cost. As we live longer, more of us are going to get Alzheimer's disease. In the United States alone, there are five million people suffering from Alzheimer's. And these diseases last for years. When you consider the emotional and economic costs to the individual and their families, our country can't sustain this health-care problem.

We haven't found solutions using conventional approaches, so working on protein folding in yeast is an out-of-the box idea, but it's not based on fantasy. It's based on highly conserved, ancient processes and the fact that yeast provides a discovery platform that you can move in much more quickly than you can in other cells and tissues.

Richard Sever: Just as Randy Schekman looked in yeast for secretory pathways because they were conserved, and Paul Nurse did it for the cell cycle, your notion is that the processes in cells in Alzheimer's and Parkinson's patients are fundamental and will be recapitulated in yeast.

Dr. Lindquist: Right. At least in terms of the initiating pathologies, what happens is that these proteins are going to the wrong places and misfolding. Of course, there are complex aspects of the biology that can only be recapitulated in a living nervous system, but the aspects that can be studied in yeast are quite broad. We've clarified a lot of murky factors about the genetics and we've found chemical compounds that rescue not only yeast but also human neurons.

Richard Sever: What do you do to the yeast cells to get them to mimic the pathology?

Dr. Lindquist: It's very simple. There are proteins in human beings that are prone to misfolding and are found in big, globby aggregates in the brain. For many years, no one was sure if those globs were causative or if they were just a consequence of other pathologies. What's become clear is that they are intimately involved in the causation of the diseases: proteins like amyloid- $\beta$ in Alzheimer's, TDP-43 (transactive response DNA binding protein) for amyotrophic lateral sclerosis, $\alpha$-synuclein for Parkinson's and multiple system atrophy. We take the coding regions for these proteins and link them to a promoter that we can turn on or off in a yeast cell. We can transform the cells and manipulate them while they're still healthy. When we want to study what's going to go wrong, we switch them into a different medium that turns on the promoter. Every cell expresses the inserted gene at exactly the same time in exactly the same way, so you get this incredibly rapid and synchronized onset of the pathology. The first thing we did was look genetically at what would rescue the cells because at that stage you could've just been looking at nonspecific protein aggregation. It might've been interesting, but not really anything disease-specific that would make a difference to neurons.

Richard Sever: If you look at amyloid- $\beta$ as opposed to amyloid- $\tau$ or Huntington or $\alpha$-synuclein, do you see different things happening?

Dr. Lindquist: Completely different genes will rescue them and we get different phenotypes. For example, vesicles are little packages that contain things like neurotransmitters in neurons or mating factors in yeast. These are made in the endoplasmic reticulum and are, through a series of complex arrangements, secreted from the cell. 
Then another cell takes up the signal and responds. That's a process that's very highly conserved. We find that for both amyloid- $\beta$ and for $\alpha$-synuclein, aspects of vesicle trafficking are disturbed, but they're different ones, and Huntington and TDP-43 are completely different from both of those.

Of course, we had all these interesting genetic results in yeast but didn't know if they would matter to a neuron. Then we made two discoveries. One was that when we expressed the genes that rescued yeast cells in neurons from a nematode, or in rat cortical neurons, or even in human neurons, they rescued those nerve cells from the phenotypes that are precipitated by those pathological proteins. The other factor was that, as the genetics on human disease got better and we understood more about the proteins that, when mutated, are contributing factors, we compared the yeast and human gene sequences and found a lot of matches. And it works both ways. We get some information from human genetic studies and show that they work in yeast, and the yeast allow us to understand the biology better. Then we go back to the human cells and find that the biology we discovered in yeast plays out there as well.

Richard Sever: You're doing some of your modeling computationally. Can you talk a bit about that?

Dr. Lindquist: There are certain features about the biology of yeast that make them unbelievably good for genetics. As a result there have been tens of thousands of genome-wide screens done in yeast, which means that when we perturb the system we can search every gene in the genome to see how it affects them. This has created a dense, connective network of genes we can tap into. You can make genetic maps where we connect the genes functionally and then use sophisticated programs to find homologs of the yeast genes in human cells. We can do amino acid sequence matching, and there also are programs based on threading structures of proteins onto previously known structures from human cells. There's also something called neighborhood analysis that relies on an understanding of protein-protein interaction networks. Our understanding of gene relationships in humans is very primitive now, but there's a lot of protein-protein interaction knowledge about both humans and yeast. You might not be sure if a particular sequence is homologous or not, but if two of the other genes it connects to in yeast are clearly homologs of two genes in humans, then, yes, the sequence you're interested in is probably homologous as well. That allowed us to create computational maps, transposing well-understood yeast relationships onto human genetic networks and connect those genes in meaningful relationships.

We found, for example, that there were genes implicated through genetic analysis to Parkinson's disease that didn't seem to have much to do with each other. People wondered if they just happened to damage dopaminergic neurons and therefore gave a Parkinsonian phenotype but weren't otherwise related. In yeast, we can see the connections because when we overexpress $\alpha$-synuclein, all the genes that are involved in the human disease can rescue, even though they're in different trafficking pathways, or play a role in metal ion homeostasis, or in calcium signaling.

Richard Sever: Have there been other surprises?

Dr. Lindquist: We've been able to connect genes that have no homologs in yeast. Mutations in a gene called $L R R K 2$ kinase (leucine-rich repeat kinase 2) are a common cause of Parkinson's disease and there's absolutely no homolog in yeast. It turns out to have a later-evolved function in humans. It didn't slot into an empty space though. It evolved to work within a network that was already there. The network it interacts with is present in yeast even though the kinase isn't, so we could figure out where it was mapping and what it was interacting with by relating it in that way.

Richard Sever: You're also using this as a discovery system for small molecules that might help the treatment.

Dr. Lindquist: Yes, and that's one of the aspects I'm potentially most excited about. Because yeast cells are so easy to grow and manipulate, we were able to screen more than 500,000 compounds for the ability to rescue yeast cells from $\alpha$-synuclein toxicity. We're still only beginning to test if the rescuing compounds can save neurons, but we've looked at two of these compounds exhaustively and they work beautifully. The key here is that these are phenotypic screens. We looked for things that would make the yeast cells better without trying to do a target-based screen on isolated proteins. These diseases are so complex and there are such webs of interactions, we thought we'd let the cells tell us what makes them feel better.

Phenotypic screening has returned some wonderful compounds in the pharmaceutical industry. They don't use it very often because it's hard to figure out what a compound is doing, so it's hard to run a clinical trial on it. Yeast genetics solve that problem. Once we found compounds that rescued human neurons from patients with Parkinson's disease, we could go back to yeast and use three different types of genome-wide screens to find the target. You can't do that kind of screening in any other organism right now.

One of the compounds we found activates a ubiquitin ligase, an enzyme that controls vesicle trafficking. $\alpha$-synuclein blocks vesicle trafficking; our compound activates vesicle trafficking, so it counteracts the toxicity. From highly conserved protein maps we were able to show that the ligase is in human cells, too, and the compound works by the exact same mechanism. It's an entirely different way of doing drug discovery for complex diseases. 


\section{$\$_{\text {CSH }}^{\infty}$ Cold Spring Harbor Symposia SYMPOSIA On Quantitative Biology}

\section{A Conversation with Susan Lindquist}

Cold Spring Harb Symp Quant Biol 2015 80: 332-333

Access the most recent version at doi:10.1101/sqb.2015.80.030114

\section{License}

Email Alerting Receive free email alerts when new articles cite this article - sign up in Service the box at the top right corner of the article or click here. 